Int. J. Dev. Biol. 53: 1291-1303 (2009)

doi: $10.1387 / \mathrm{ijdb} .072459 \mathrm{ag}$

\title{
The cellular and genetic bases of organ size and shape in Drosophila ${ }^{*}$
}

\author{
ANTONIO GARCÍA-BELLIDO* \\ Centro de Biología Molecular "Severo Ochoa", Consejo Superior de Investigaciones Científicas, \\ Universidad Autónoma de Madrid, Madrid, Spain
}

\begin{abstract}
In this essay, we discuss the contribution of local cell interactions, as opposed to global cues, such as hormones and morphogens, to the determination of the size and shape of organs. This internalistic notion is based on the integration of physiological experiments, clonal analysis and genetic mosaics of morphogenetic mutants. Physiological experiments such as regeneration, cell dissociation and re-aggregation, and feeder layers, reveal the existence of regional specificities related to regional patterning, positional values and cell polarity. On the other hand, clonal analysis shows homogeneous and exponential growth by mitosis associated with oriented cell divisions along two axes of growth. Preferential mitotic orientations are also regional characteristic. Finally, genetic mosaics of morphogenetic mutants help to understand the genetic and eventually the molecular bases of morphogenesis, revealing the modes of cell behavior (such as positional accommodation, mitotic orientations, cell affinities and cell recognition) at work in the generation of the constant sizes and shapes of Drosophila imaginal structures. These classical and recent findings contribute to a more updated Entelechia model of morphogenesis.
\end{abstract}

KEY WORDS: regeneration, cell recognition, genetic mosaics, mitotic orientation

\section{Introduction}

The internalistic and externalistic views of morphogenesis

Causal explanations of many processes in biology have focused on either internalistic or externalistic approaches. Such is the case of heredity vs. environment; immune response, inducible enzymes and hormones vs. competent tissues and local vs. global control in morphogenesis. In general, the main question is where the instructive components of the resulting specificity reside. In an externalistic interpretation, the instructive signals come from diffusible morphogens to which naïve cells respond in a concentration depending manner. This interpretation ("positional information", Wolpert, 1969; Kerszberg and Wolpert, 2007) has been applied to pattern formation in which cells respond to outside signals independently of their neighbors. According to this view, patterns are size-invariant. In contrast, according to an internalistic approach, local cell interactions coordinate neighboring cells to proliferate, and by extension, the whole population acquires finite dimensions. The internalistic interpretation which we present herein deals with problems related to the control of size and form. It represents a model of informational position, rather than "positional information" in which it is the position of the cell which determines the behavior of its neighbors, this being the essence of the Entelechia model (García-Bellido and GarcíaBellido, 1998). In this essay, we will explore the "black box" of the responding developmental system, and its internal structure, i.e. how differential gene activity, in quality and quantity, locally determines cell behavior and hence species-specific sizes and shapes.

\section{Cell proliferation parameters}

\section{Growth and final size are disc-autonomous}

Maximal growth can be restricted under conditions of premature metamorphosis or in mutants with impoverished nutritional conditions. Continuation of growth beyond maximal size can be

Abbreviations used in this paper: AEL, after egg laying; A/P, anterior/posterior; $\mathrm{D} / \mathrm{V}$, dorso/ventral; P-D, proximo-distal.

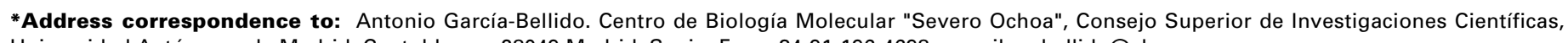
Universidad Autónoma de Madrid, Cantoblanco, 28049 Madrid, Spain. Fax: +34-91-196-4698. e-mail: agbellido@cbm.uam.es

\#Note: I would like to dedicate this essay to François Jacob, in admiration for his internalistic explanation of biological adaptation. 

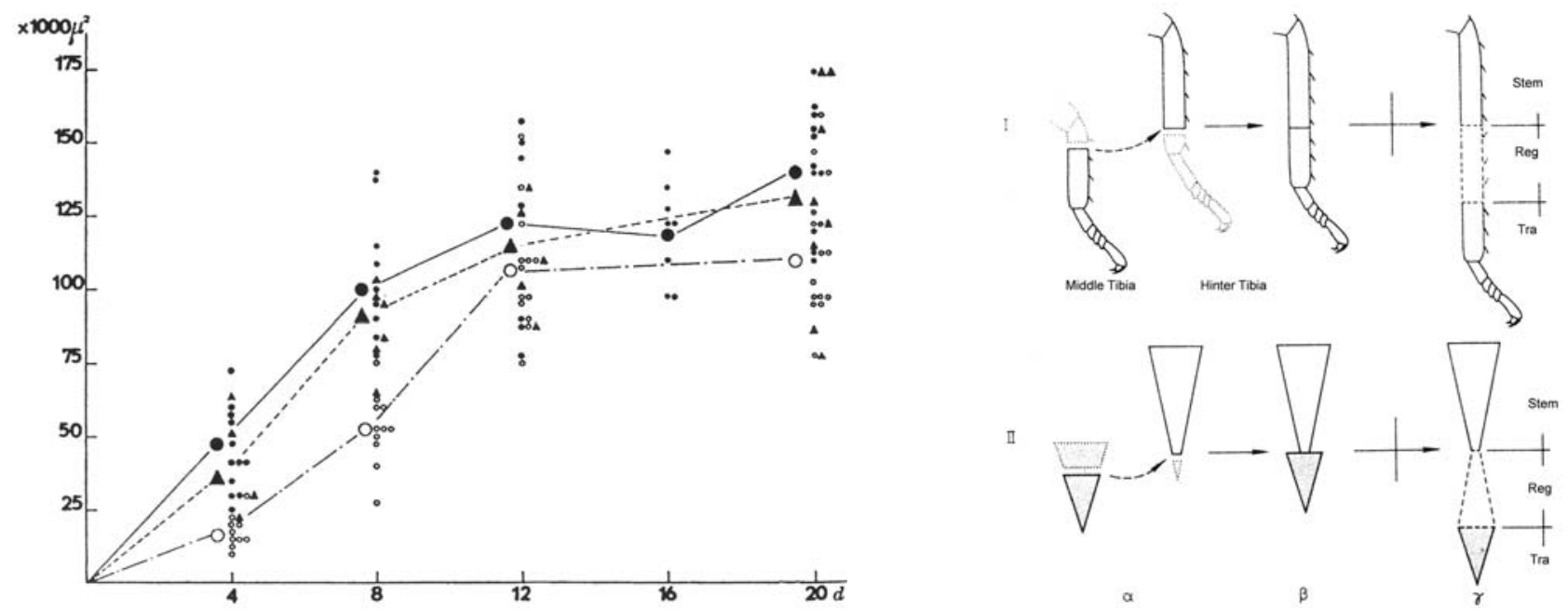

Fig. 1 (Left). Autonomous growth of 1st instar wing discs cultured in adult hosts. $\bullet$ Mated females; $\mathbf{\Lambda}$ mated females with implanted ring gland of mature larvae; $\bigcirc$ virgin females. The growth curves plateau at the volume of mature discs (From García-Bellido, 1965).

Fig. 2 (Right). Intercalary regeneration between positionally overlapping fragments of leg segments of a Leucophaea larva. The proximal stem is from the hinter leg tibia; the distal from the middle leg tibia, in order to identify the cell origin of the regenerate. (A) Scheme of the operation. (B) A gradient model of interpretation (from Bohn, 1970).

attained, up to a limit, in mutants with an altered metabolism, as observed in mutants of members of the insulin pathway (see Mirth and Riddiford, 2007). Experiments of transplantation of young (first instar) imaginal discs to adult hosts performed over 40 years ago did show that, irrespective of the hormonal and nutritional conditions of the host, the transplanted discs soon reach a maximal size which corresponds to that of mature larvae (Fig. 1) (García-Bellido, 1965). This autonomy extends to individual regions of the discs in Minutemosaics, irrespective of cell proliferation rate (García-Bellido et al., 1973).

\section{Positional values in regenerates}

Many classical experiments of appendage regeneration in insects have revealed the operational relevance of positional values in growth. In particular, the various outstanding experiments of Bohn (1970) in Blattariahave shown that the place of cut along the legs determines the amount of growth of the blastema. Terminal regeneration occurs after cuts to any leg segment in any position. However, when proximal and distal cuts are apposed, the behavior of the resulting construct differs, depending on the level of the cut. Grafts of homologous levels in different leg segments (e.g. femur and tibia) do not elicit regeneration. When there is a positional gap between stem and graft, the gap is regenerated. When proximal cuts of the graft are apposed to distal

Fig. 3. Pattern reconstruction by dissociated cells following reaggregation and culture in adult hosts. The cells derived from male foreleg discs are genetically marked by yellow and ebony. (A) The section corresponds to the tibia and basitarsus. (B) The implant itself after metamorphosis, showing the mosaic pattern. (C) A two dimensional projection of (B). Arrows are at the base of the chaetae and orientation of bracts indicates the planar polarity of the bristle mother cells inducing the bract, sometimes made up of cells of different genotypes. I and II are tibia repeats; III is the basitarsus with sex comb (modified from García-Bellido, 1966). cuts of the stem of the same leg segment, i.e. the levels of the cut overlap, then regeneration, instead of stopping, continues generating intermediate duplications with opposite polarity, as judged by the orientation of cuticular elements of the pattern (Fig. 2). Cells of both stem and graft contribute to the intercalar regenerate. Clearly, the confrontation of distal and proximal located cells drive the proliferation of the regenerate until the landscape of positional values is smoothly continuous. This extra growth can be considered as "accommodation growth"(see below). Similar outgrowths occur when stem and graft differ in circular values (as in left to right leg transplantations). Proliferation is then driven by differences in positional values along two axes, proximo-distal ( $P$ $D)$ and anterior/posterior $(A / P)$, rather than by different concentra-

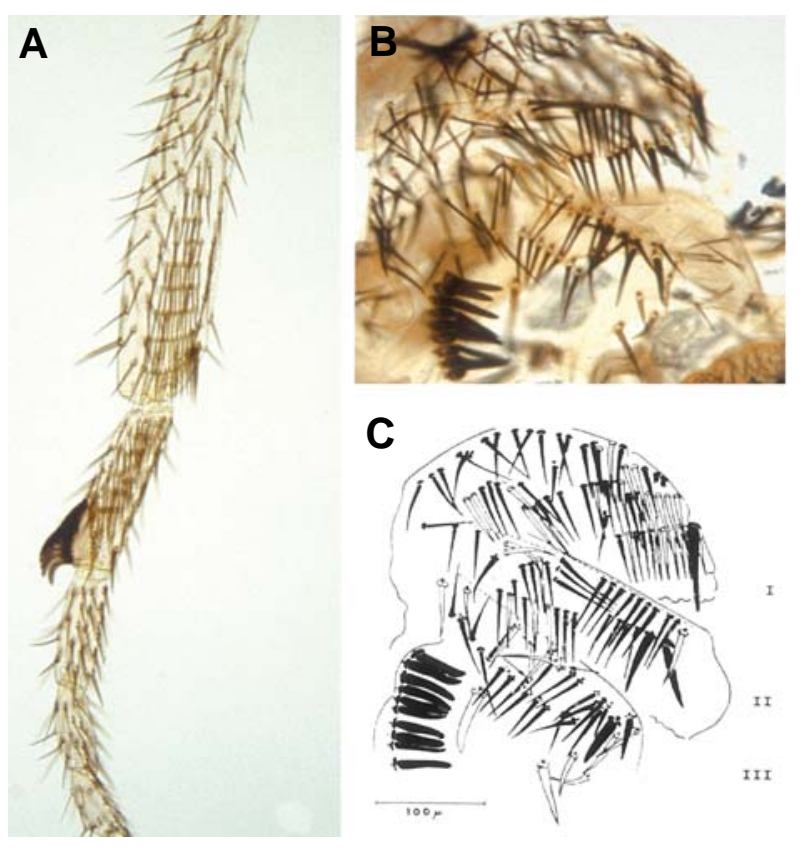




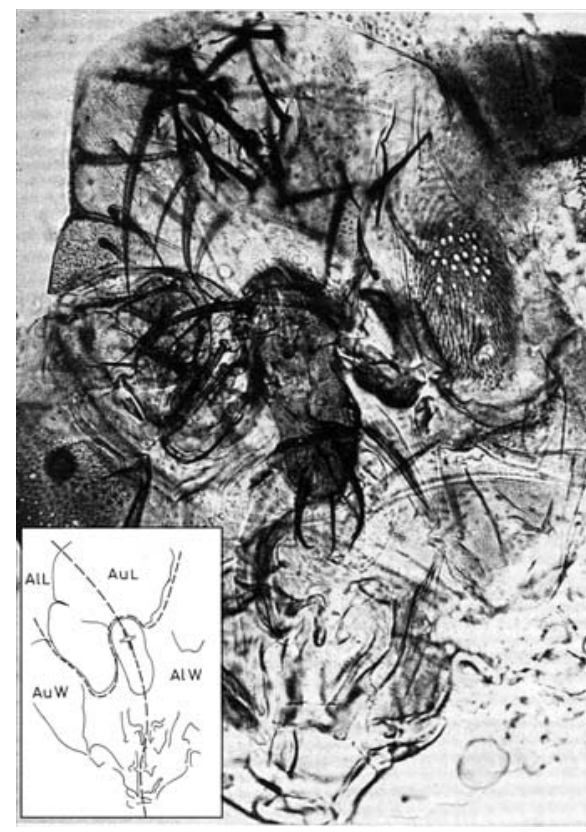

Fig. 4. Pattern duplications in aggregates following transdetermination of leg (L) to wing (W) and vice versa. Wing cells (yellow and multiple wing hairs) were dissociated and mixed with leg (ebony) cells and cultured for 20 days in adult hosts. After metamorphosis autotypic (Au) and Allotypic (Al) structures of leg and wing appear duplicated in mirror image along the line indicated in insert. (From GarcíaBellido, 1972).

tions of morphogens emanating from the blastema or from compartment borders. What are the molecular bases of these positional values?

\section{Cell recognition and positional values}

In regenerates, the blastema cells grow and organize themselves in an intact territorial context. Following cell dissociation, we can ascertain the specific information that individual cells may carry (García-Bellido, 1966). Dissociated and aggregated cells of grown imaginal discs can be cultured in adult hosts from hours to days and then transplanted to larvae to undergo metamorphosis and cuticular differentiation. In re-aggregates of cells from different imaginal discs, which are genetically marked, leg and wing cells sort out and differentiate in patterns typical of the disc of origin. Re-aggregates of cells of the same disc but of different regions also sort out. However, they form mosaic patterns when derived from the same region of the disc (Fig. 3). These patterns are, albeit incomplete, normal with different elements organized in mosaic fashion. Single cells may appear with abnormal polarity relative to other elements of the pattern. Thus, dissociated cells carry positional cues and polarity which are characteristic of the region of the disc from which they derive. This information is used to allocate the cells correctly in the re-aggregate pattern. Cell recognition is based on position rather than on the cell type of the pattern element. These positional values are still manifested in the cell membrane after cell dissociation. Aggregates cultured for several days lead to more complete patterns, possibly due to intercalary, local regeneration without further global references. We will see later how proliferating cells may use their positional values during normal development.

\section{Pattern formation by the offspring of isolated cells}

Feeder layer experiments allow us to ascertain the heredity of determination of single cells in mixtures. These mixtures consist of heavily irradiated cells of one type of genetically marked imaginal disc and single untreated wild type cells of another type. The irradiated substratum prevents growth competition and supplies a support for the proliferation of the single cells (GarcíaBellido and Nöthiger, 1976). The offspring of single cells retain their determination to belong to e.g. anterior or posterior compartments. They may form incomplete but well-formed patterns. Pattern expansion does not depend on the nature of the disc origin of the feeder layer. Proliferation patterns may include D/V elements of the wing margin. Their frequency is too high to have derived from incomplete dissociation, and they are possibly formed de novo. Thus, individual cells can proliferate to generate morphogenetic patterns without signals coming from global organizers.

Following fragmentation and regeneration, or cell dissociation and re-aggregation, single cells or a few cells may change their original determination (the so-called "transdetermination", Hadorn, 1978 ) in culture. Thus, leg cells may transdetermine to wing cells and vice versa, changing their cell affinities along with the histotype (García-Bellido, 1966, 1972). In mixtures of leg and wing cells,

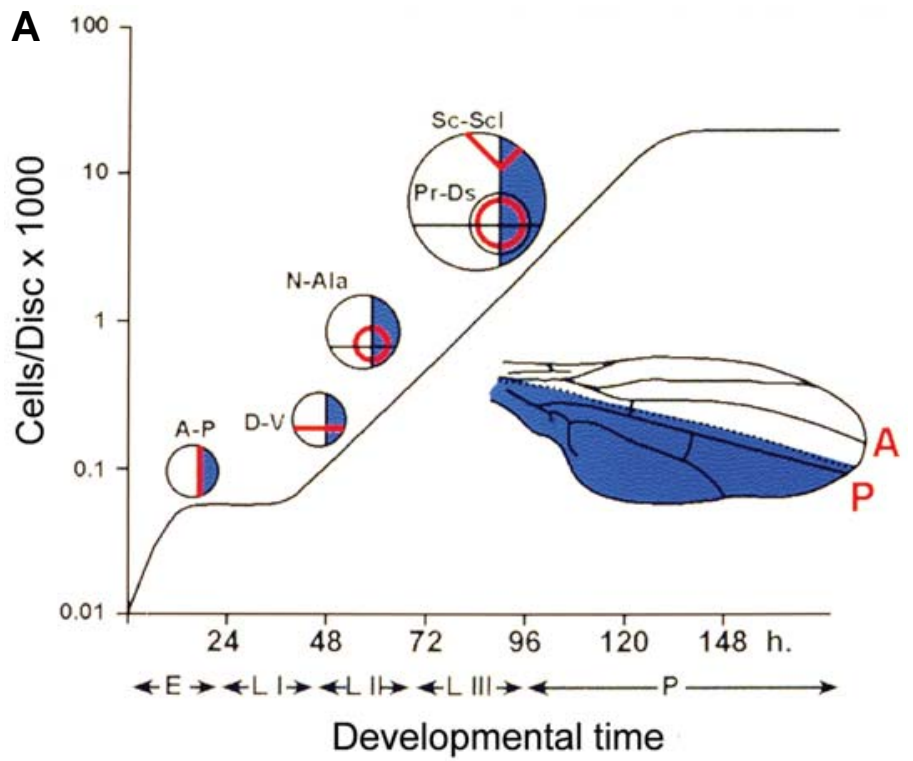

B
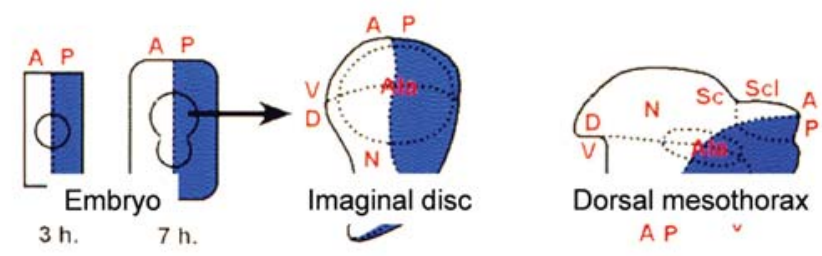

Fig. 5. Clonal restrictions separating compartments during the development of the wing disc. In blue the posterior compartment (en expression). (A) Topological subdivisions in successive compartmentalizations (A-P, anterior-posterior; D-V, dorsoventral; N-W, notum ala; PrDs, proximodistal; Sc-Scl, scutum-scutelum. Exponential curve of growth illustrates number of cells with developmental time and larval stages. (B) The topographical representation in the disc and in the adult. (Modified from García-Bellido et al. 1973). 


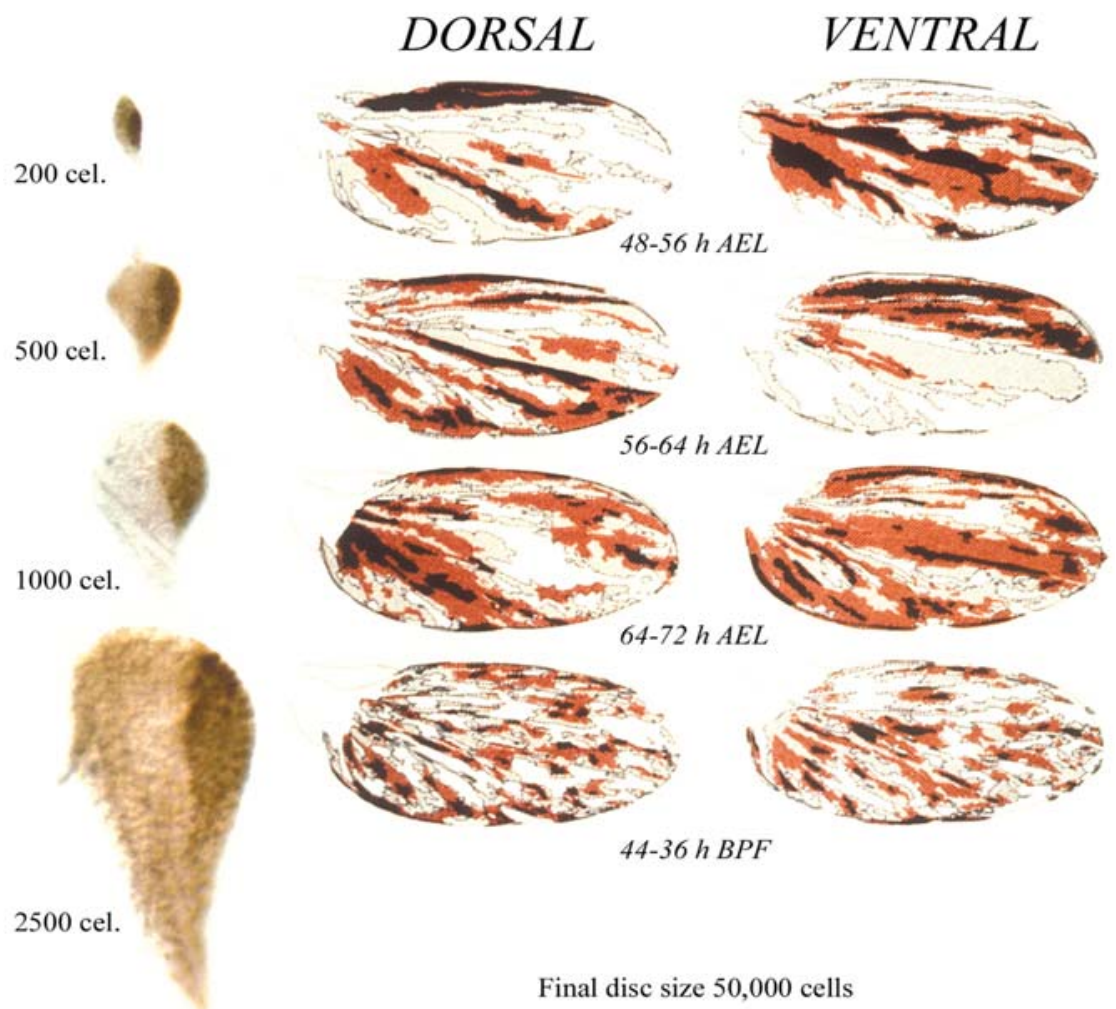

Fig. 6. Changing frequency distribution of clones initiated at different times, wing surfaces and regions. To the left, the relative size of the disc at different ages in $h A E L$ (after egg laying) or BPF (before puparium operation). Posterior compartments labeled with engrailed expression. (From González-Gaitán et al., 1994).

transdetermined (allotypic) wing cells may encounter their autotypic partners, and the same applies to leg transdetermined cells. In such cases, the allotypic and autotypic cells can either differentiate into mosaic patterns, or may copy the autotypic patterns then encounter in mirror-image symmetry (Fig. 4) along any virtual line of the pattern. We do not know how this merging or copying of patterns comes about, but clearly, cell
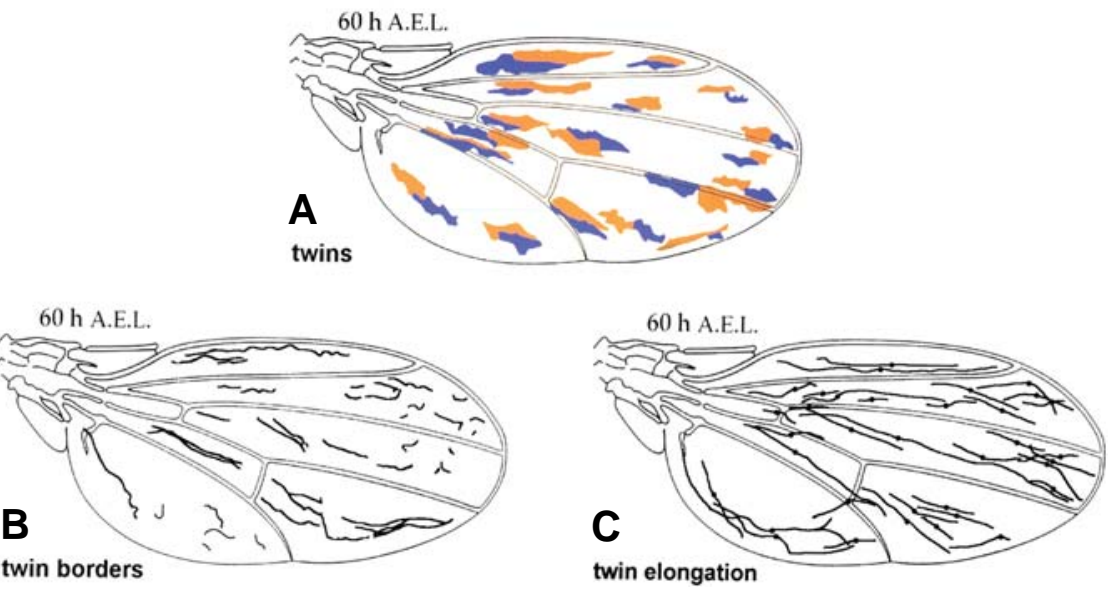

Fig. 7. Analysis of twin clones allows us to ascertain the topological location of their mother cells. (A) Twin clones. (B) Borders separating twin clones. (C) Mother cells as dots separating twin clones and their elongation. Notice how elongation lines may overlap, indicating intercalar growth throughout the primordium. (Resino et al., 2002). AEL, after egg laying. recognition and new references for patterning and growth are at work. In the first case of integration in a mosaic fashion, cell recognition and intercalary regeneration may play a role. In the case of pattern copying along a symmetry line, opposite cell polarity of the autotypic blastema may encounter an opposite polarity in the transdetermined cells, leading to the generation of a symmetric axis, following normal growth away from this axis. In any case, this occurs independently of any known global reference from the normal growing disc.

\section{Clonal growth}

Induced mitotic recombination can give rise to single clones or twin clones of genetically marked cells which continue to proliferate and can be visualized in either the imaginal disc or the adult, as spots in a mosaic with surrounding wild type cells. The study of these clones reveals numerical and qualitative parameters of disc growth (García-Bellido and Merriam, 1971). The wing imaginal disc is defined slightly after blastoderm formation from 20-30 founder cells. Cell proliferation, after disc formation, starts at the end of the first larval instar and continues until 20 hours after puparium formation with about 50,000 cells $(30,000$ for the wing), after which follows cuticular differentiation. The imaginal disc events which occur in early pupae to attain the final shape and size of the adult mesonotum and wing proper are illustrated in Fig. 5.

Proliferating cells in the disc show clonal restrictions which separate contiguous compartments. As shown in Fig. 5, the first restriction separates the $A$ and $P$ cell populations (compartments) already in the blastoderm. Subsequent restrictions during larval stages separate $\mathrm{D} / \mathrm{V}$ and notum $(\mathrm{N})$ / wing $(\mathrm{W})$ compartments. Interestingly enough, in the wing blade, the $D /$ $\mathrm{V}$, and $\mathrm{A} / \mathrm{P}$ compartments show proliferation patterns of growth, which are symmetrical at both sides of their borders (García-Bellido et al., 1973). Other clonal restrictions appear later; along the longitudinal veins generating new symmetries in intervein regions. Compartments are poly-clonal in origin, but, except for compartment boundaries, proliferation is indeterminate (Fig. 6). However, clone shape and mitotic orientation (its main growth axis) is a characteristic feature of compartments of the wing disc and its regions (Figs. 6,7). Later in development, specific cell lineages appear related to particular histotypes, wing margin, longitudinal veins, etc. (Fig. 8).

Clone size increases exponentially and homogeneously in all disc regions. Early-induced clones give rise to large territories and are reduced to one single cell in mature discs, when the number of clones is of the order of several hundreds. Clones are compact, that is, the cell 
A

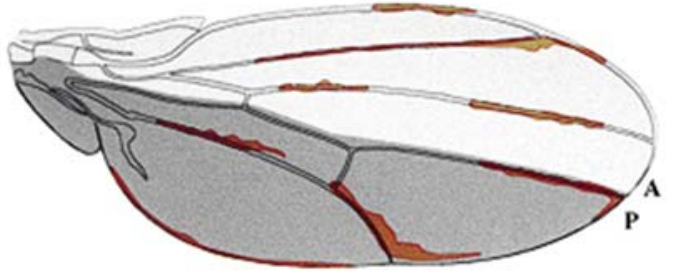

B
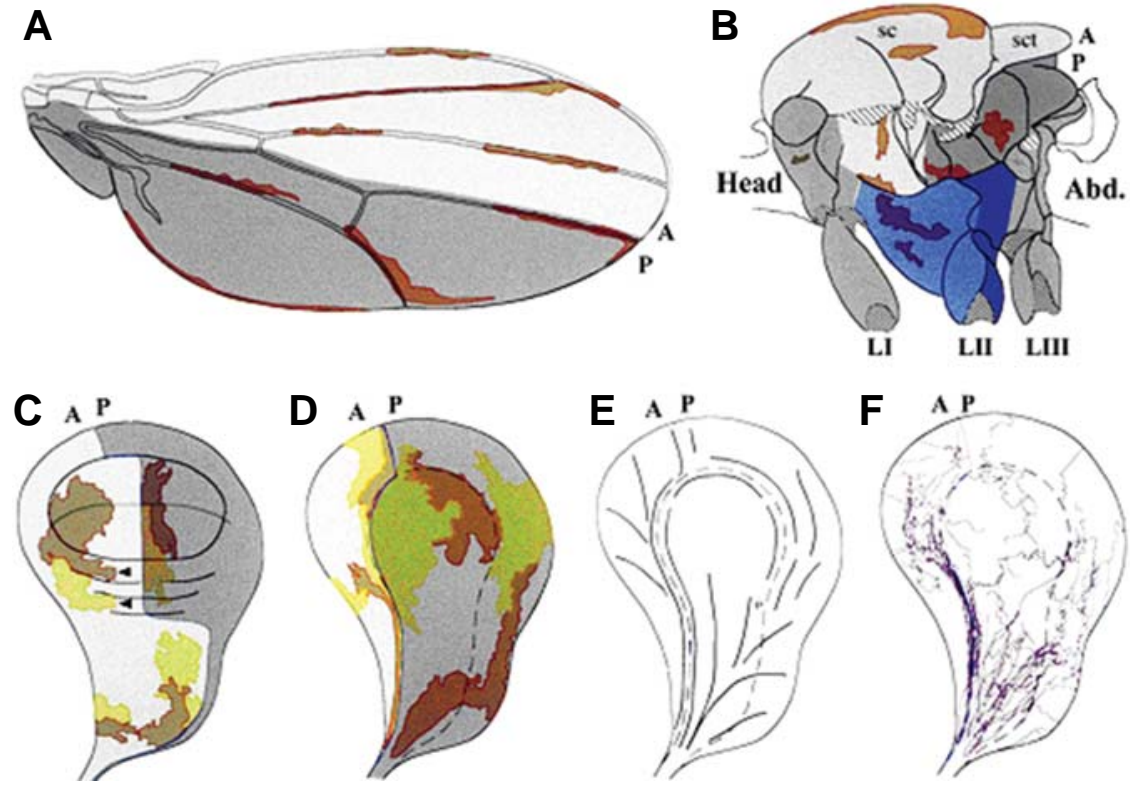

Fig. 8. Twin clones in imaginal wing discs (dorso, ventral) and in the adult $(A, B)$ showing region characteristic shapes. In $(\mathbf{E})$ and $(\mathbf{F})$, preferential clonal orientation and clone outlines in a ventral view of the disc (from Resino et al., 2002).

offspring remain together. Analysis of twin clones allows us to ascertain the topological location of the mother cell in the interface between the two twin clones and thus define their growth directions (Resino et al., 2002). Mother cells can be anywhere in the disc. The resulting daughter clones grow away from the location of the mother cell proximo-distally or anteriorposteriorly to it (Fig. 6). Cell proliferation is then intercalary and the mother cells in any clone are located topologically in the center of the clone. Offspring cells of the original mother cell acquire in successive divisions their positional values away from the mother cell along both axes of growth. In the wing blade, early clones are mainly elongated along the P-D axis; later the clones widen. In the legs, early clones appear as elongated ribbons, over several leg segments (say from coxa to tarsus) without encountering clonal restrictions besides the A/P one. In the notum, early clones are fundamentally isodiametric. The shape of clones, as visualized in the imaginal disc, remains in the adult after disc eversion in metamorphosis. Thus, the shape of the adult organ directly reflects the shape of the clones (Milan et al., 1996). Under normal conditions there is no localized cell mortality that could contribute to local differences in form (Milán et al., 1997).

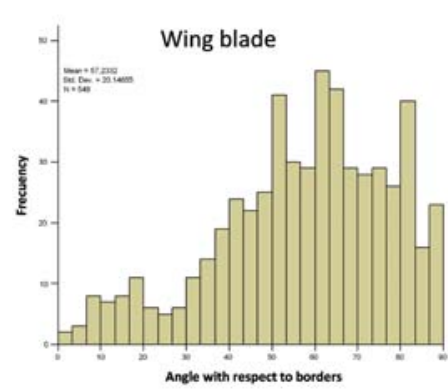

The direct observation of proliferating cells in the imaginal disc reveals several features relevant to morphogenesis. Cell divisions in the disc are not randomly distributed. Clusters of two - five synchronic cells appear along development in variable positions. These correlate with the changing clustering of cells in G2 in different compartments and regions (Fig. 6). Thus, although average cell cycling lasts around eight and a half hours, there are local variations. Clusters of synchronic cells in early and late $\mathrm{S}$, in G2, anaphase and mitosis appear along with islands of cells in $\mathrm{G} 1$ that remain for more than 16 hours in this stage (Milan et al., 1996). These synchronic clusters are not clonally related. Synchronic clusters seem to move along the wing blade in waves (see Fig. 7 in Milan et al., 1996). Interestingly, the mitotic orientations of members of these clusters are mainly parallel to each other (see below) indicating coordinated local cell interactions releasing cell divisions.

The analysis of telophases and anaphases reveals mitotic orientation, which are characteristic of the wing region (Baena-López et al., 2005). Thus, they appear parallel to the wing margin in a narrow band along the $\mathrm{D} / \mathrm{V}$ border and orthogonal to it in central regions of the wing blade (Fig. 9). However, mitotic orientations are more random in the notum and in pleural regions. The relative orientation of postmitotic cells follows that of the preceding mitotic orientation (Fig. 12). Thus the local shape of clones
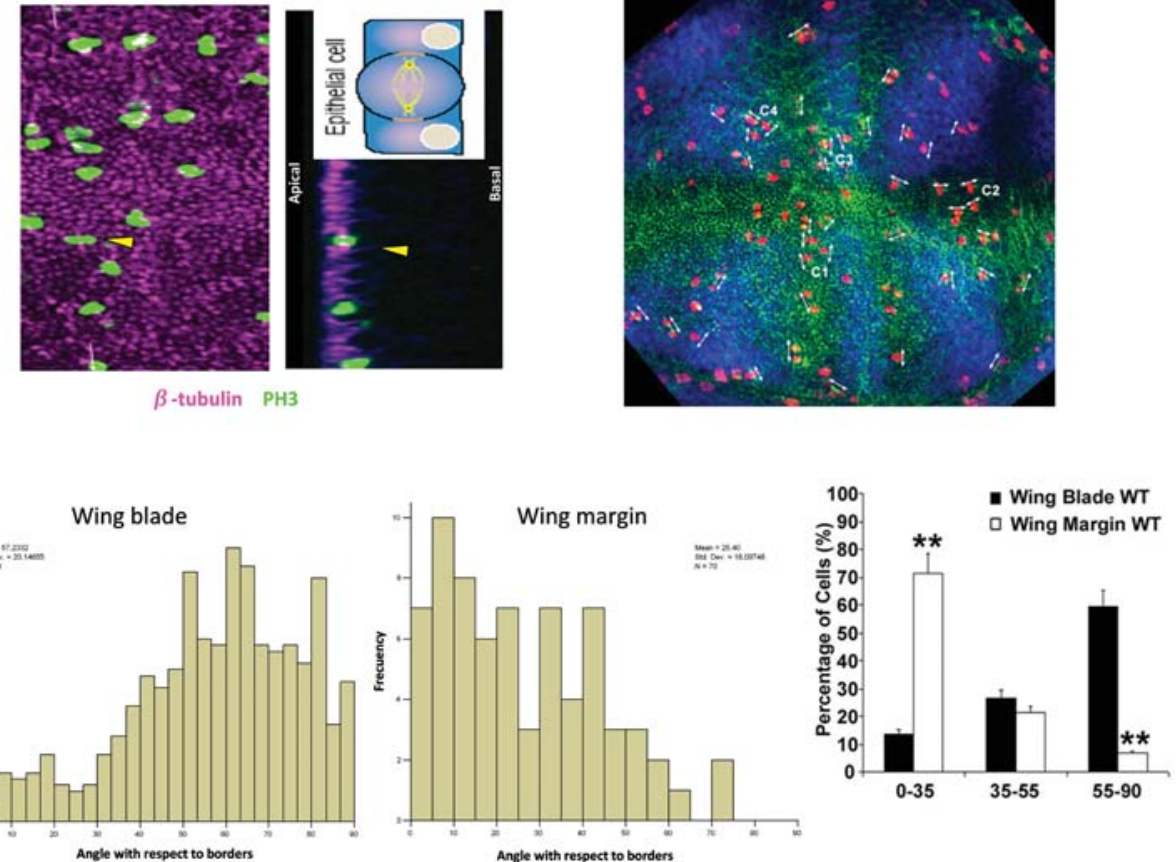

Fig. 9. Mitotic spindle orientation in mature wing imaginal discs. Differential prevalent orientations along the wing margin and in the wing blade. $\beta$ tubulin labels for the mitotic spindle and ph3, phosphohistone 3 the chromosomes. 1-4; clusters of cells. 0-90 parallel to or perpendicular in 90 degrees, to the wing margin (from Baena-López et al., 2005). 

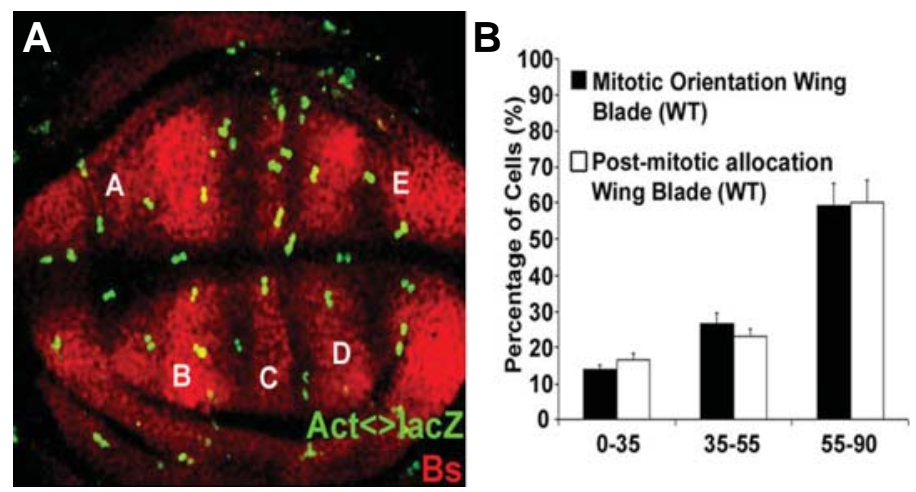

Fig. 10. Spindle figures in a dorsal view in a mature wing disc. (A) Postmitotic daughter cells retain the orientation of mitotic cells labeled by flipout of lacZ. Bs, bristle labels the intervein regions. (B) Groups of angular differences with respect to wing margin: parallel or $90^{\circ}$ perpendicular to it.

reflects the alternating orientations of the mitotic spindles, and therefore determines the final shape of final organs and appendages in their distinct regions.

\section{Genetic control of size and shape}

Control of growth in genetic mosaics for compartment selector genes

In genetic mosaics, there is a confrontation of mutants and wild type territories where problems of cell autonomy for mutant as well as confronting territory can be analyzed. The engrailed (en) mutation causes the homeotic transformation of posterior compartment into anterior one of the same segment (GarcíaBellido and Santamaría, 1972). In mosaics, enbehaves cell autonomously (Fig. 11). Early en mutant clones, appearing in posterior

Fig. 11 (Top). Growth accommodation in genetic mosaics of $e^{-}$(C,D) and ap (E,F). Co, 1-5 costa and longitudinal veins, showing their dorsal or ventral corrugations. In (C), clones of en (def. of en and inv) in three different positions in the posterior dorsal compartment. Notice intercalar pattern duplications. In (D), effects of an en- clone most distant to the A/P boundary with extra growth in the ventral compartment. In (E), clones of ap (actually chip) in three positions in the anterior dorsal compartment. Duplication outgrowths are larger the farther away from the DN border are initiated. (F) A clone initiated close to the A/P boundary leads to maximal accommodation caused by a small (arrow) clone (F: taken from the PhD thesis of Fernández-Fúnez).

Fig. 12 (Bottom). Heterogeneous expression of $\mathbf{V g}$ in the wing blade. (A) The expression of $\mathrm{Vg}$ (yellow) and $\mathrm{Wg}$ (blue) is shown in the wing, surface view in $A$, and confocal sections of mature discs. Yellow and blue lines correspond to $\mathrm{Vg}$ and Wg expression in DN boundary and veins (2-5) (From Baena-López and García-Bellido, 2006). compartments form A/P boundaries in the clone border. Clones induced at the same time differ depending on their position. They are large when close to the A/P boundary and smaller far away from it. This is an indication of disparity in positional values. More interestingly, the wild type tissue responds by extra growth, which is larger the further away is the clone from the endogenous $A / P$ boundary (Fig. 11). These extra growths duplicate in mirror image features, such as veins, with opposite polarity to the wild type normal territories. The same applies to both dorsal and ventral mosaics. Clearly, the growth response is the result of the position of the mosaic spot, rather than of the extent of the new ectopic A/ $\mathrm{P}$ border, the putative source of morphogens (such as decapentaplegic, $d p p$ ). These accommodation effects are reminiscent of the result of experiments in legs leading to intercalary regeneration, as seen above.

We encounter a similar situation in mosaics of the selector gene apterus $(a p)$ for dorsal compartments. The appearance of a wing margin between wild type dorsal and apmutant ventral cells causes a reaction in the surrounding territories. Large clones, appearing close to the endogenous D/V border, are surrounded by less accommodation than smaller clones further away from the D/V border. These cause large outgrowths, with opposite polarity
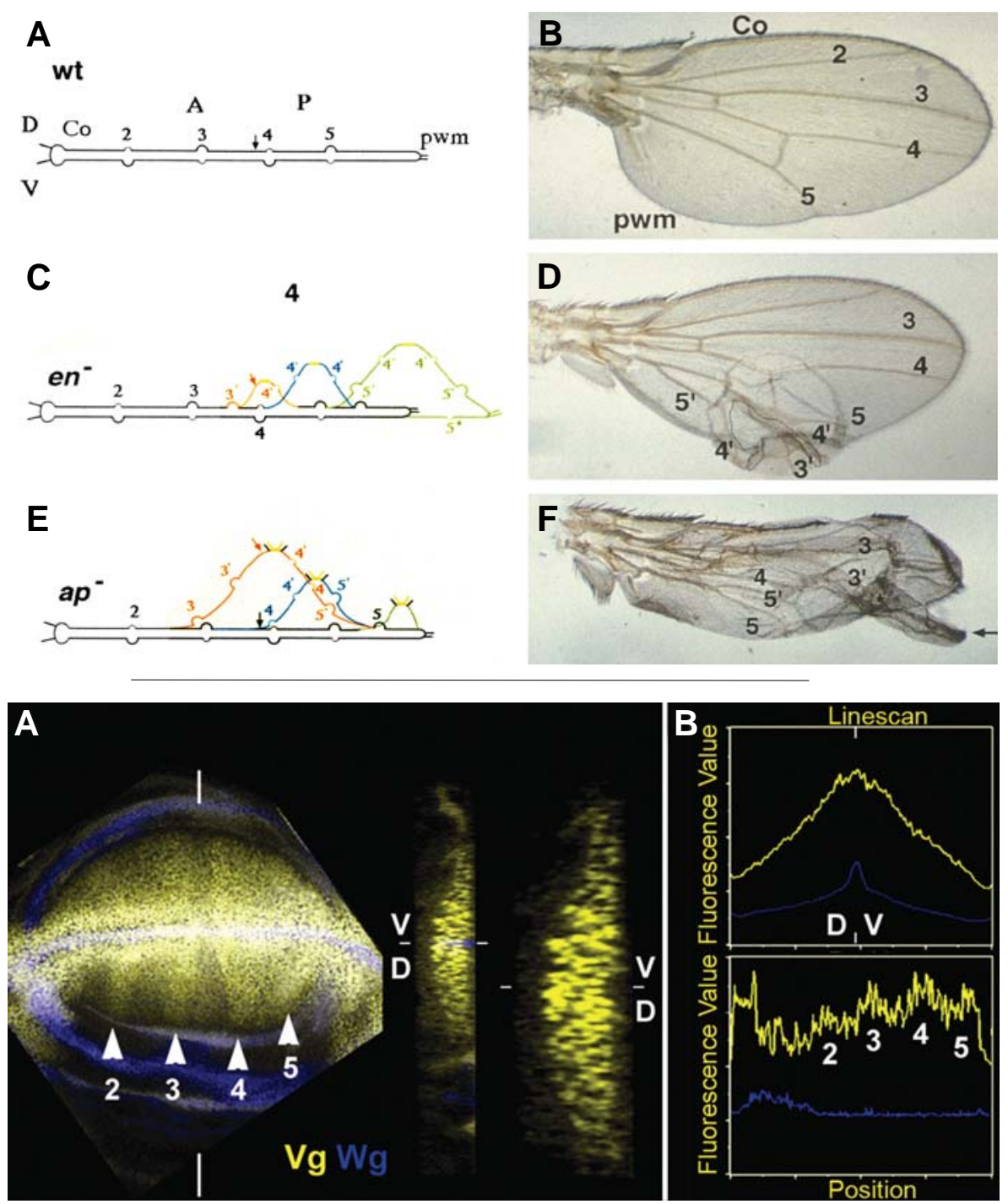


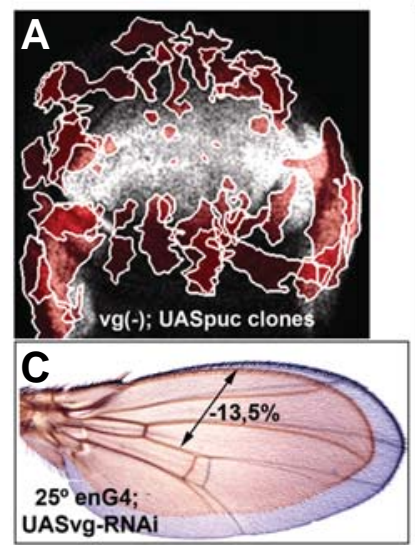

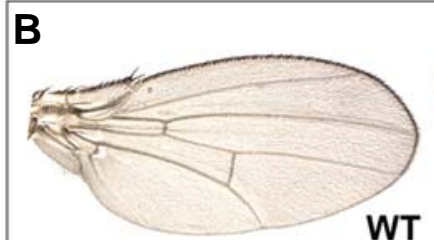

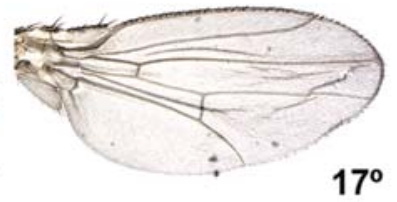

$17^{\circ}$
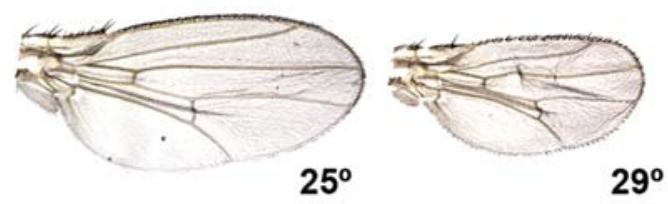

Fig. 13. Loss of function of $\mathbf{V g}$ (using a $\mathbf{V g}$ RNAi) affects autonomous and non-autonomously the growth of the wing. (A) $\mathrm{Vg}$ clones (associated to over expression of puc to prevent cell death). Notice heterogeneous size distribution depending on position, small and narrow in the wing border and large in proximal regions. (B) Reduction of size with temperature of culture. (C) Autonomous effects of $v g$ RNAi on the P compartment and non-autonomous accommodation in A compartment, at $25^{\circ}$ (from Baena-López and García-Bellido, 2006). to wild type surrounding cells (Fig. 11). This can be explained, once again, rather by positional accommodation than by the restricted amount of morphogens, such as wingless ( $w g)$, emanating from the small new ectopic D/V compartment boundary. The same accommodation responses take place in apmosaics in the anterior-dorsal compartment.

Interestingly, in both cases, $e r$ and $a p^{-}$accommodation includes growth and patterning by cells of the compartment adjacent to that of the mosaic. Thus the ectopic source of morphogens of the mosaic can override the organization dictated by the endogenous sources, it seems that is the continuity of positional values rather than the postulated morphogens what determines growth, over morphogen concentration or is independent of it.

\section{Growth in the wing blade}

Wing blade size and shape are affected by several mutations. The mutation vestigial ( $v g$ ) causes strong reduction of the wing blade. The Notch and wg signaling pathways activate the expression of $v g$. This gene encodes for a transcription factor that forms

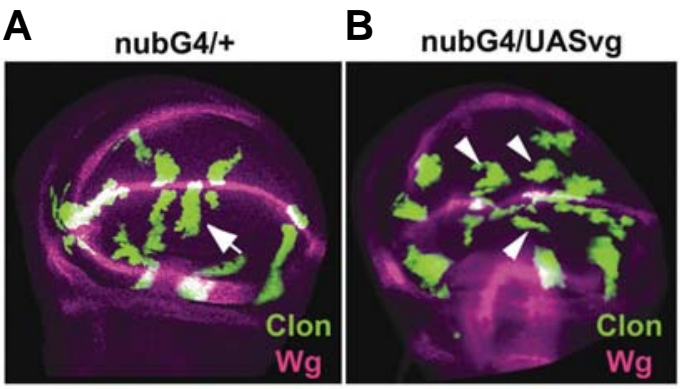

D

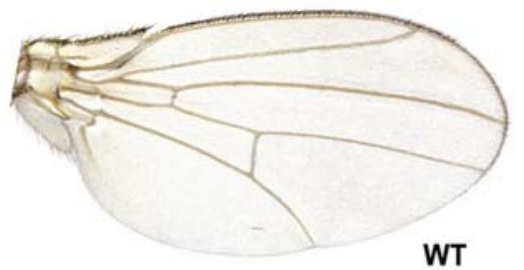

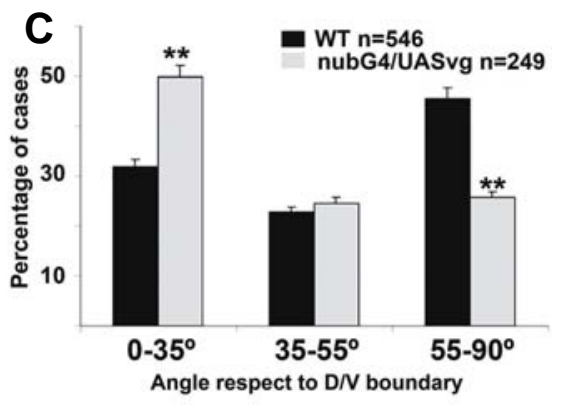

E

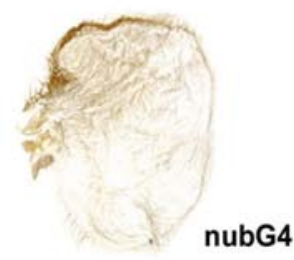

Fig. 14. Heterogeneous over expression of $\mathrm{Vg}$ modifies the shape and size of clones in a position dependent manner. (C) Changes in the orientation of cell division in the wing blade (using nub G4/UAS vg). (D) Wildtype. (E) Global effects of the same gene construct on wing shape. (From Baena-López and García-Bellido, 2006). a complex with Scalloped ( $s d$ ) that control the expression of genes such as dachsous $(d s)$ and fat $(f t)$. Fat and Ds are two protocadherins that connect cell membranes of neighboring cells (see below). The $\mathrm{Vg}$ protein expresses itself as a gradient with maximal values in the wing margin, where the gene is activated, and minimal in the bases of the wing blade (Fig. 12) (Baena-López and García-Bellido, 2006). This gradient distribution of $\mathrm{Vg}$ product does not reflect diffusion from a source but rather the distribution of heterogeneities between cells. The reduction of $v g$ expression, by driving $v g$ RNAi over the wing blade, reduces further and further the wing size with lesser $v g$ amount. So far as expected. This decrease in size is not due to cell mortality but to lower cell proliferation. Mosaic $\mathrm{vg}$ clones of the same RNAi construct show clone sizes which are smaller the closer they are to the wing margin (Baena-López and García-Bellido, 2006) (Fig. 13). In addition, they cause a non-autonomous reduction of growth (negative accommodation) in the wild type surrounding cells, close to the D/V boundary. The smaller size of $\mathrm{vg}$ mutant wings is accompanied by a change in the shape of clones, causing rounder wings (Fig. 13). The round shape is associated to random orientation of the mitotic spindle in the dividing mutant cells of the blade (Baena-López and García-Bellido, 2006).

The gain of function (GOF) condition for $v g$, using the same nubGAL 4 driver as above, can be used to homogeneously over-express $v g$ all over the wing blade (Baena-López and GarcíaBellido, 2006). Surprisingly, the result is the same as in loss of function (LOF) condition, i.e. reduced wing size, rounder wings and randomized mitotic orientations (Fig. 14). Thus, both homogeneous overexpression and homogenous insufficiency affects both cell proliferation and spindle orientation.

Contrarily, heterogeneous expression of $v g$ in mosaics causes autonomous and non-autonomous extra-cell proliferation (Fig. 15). This response varies depending on the position of the clones along the P-D axis of the wing. Outgrowths, including the mutant clone, 

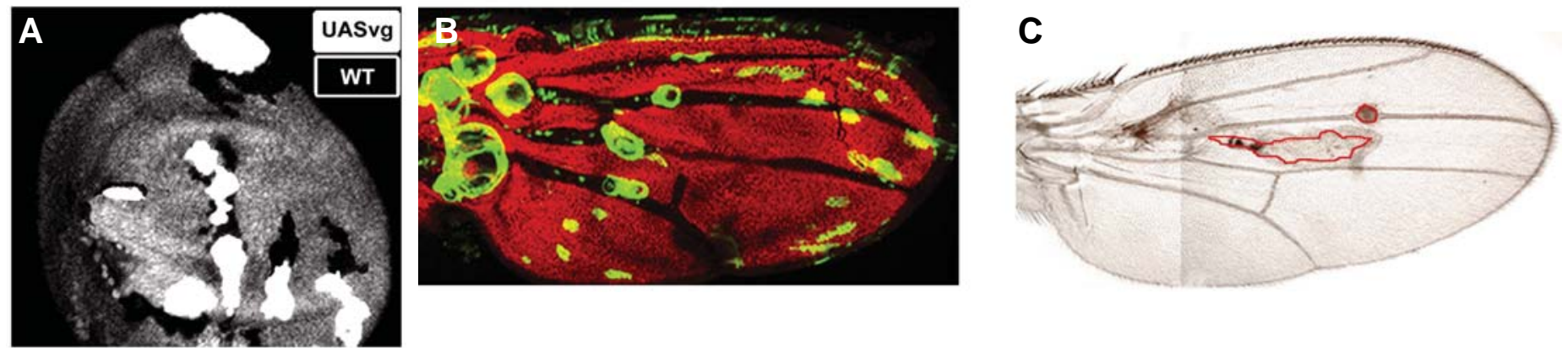

Fig. 15. Heterogeneous expression in clones of vg (and twins) in the notum wing disc (A), in prepupa (B) and in the adult wing (C). Notice in (C) that the extent (outline) of the clone is smaller than that of the outgrowth (From Baena-López and García-Bellido, 2006).

are larger the more proximally they are originated. This result points to a role of $v g$ in mosaics: discordances in positional values of highly expressing cells and surrounding low expressing cells causes accommodation in the form of extra-growth and pattern duplications. The resulting accommodation is driven by differences in positional values between neighboring cells, not by the absolute amount of vg (Baena-López and García-Bellido, 2006). The clonal over-expression of $w g$ does not cause extra-growth but when it is expressed simultaneously with $v g$, the outgrowths are larger and show distal wing margin patterns (Fig. 16). This is possibly the mechanism that elicits growth in normal development of the wing blade: differences in amount of expressions between cells along the P-D gradient led cells to divide and orient their mitoses (Fig. 17).

The gene nubbin (nub, a transcription factor) is homogeneously expressed over the whole wing blade. The LOF mutant condition of nub causes much-reduced wings with a short wing margin. In genetic mosaics the reduction of nub clone size also depends on the position; smaller clones appear the further away from the wing base. Distal clones differentiate histotypes corre-
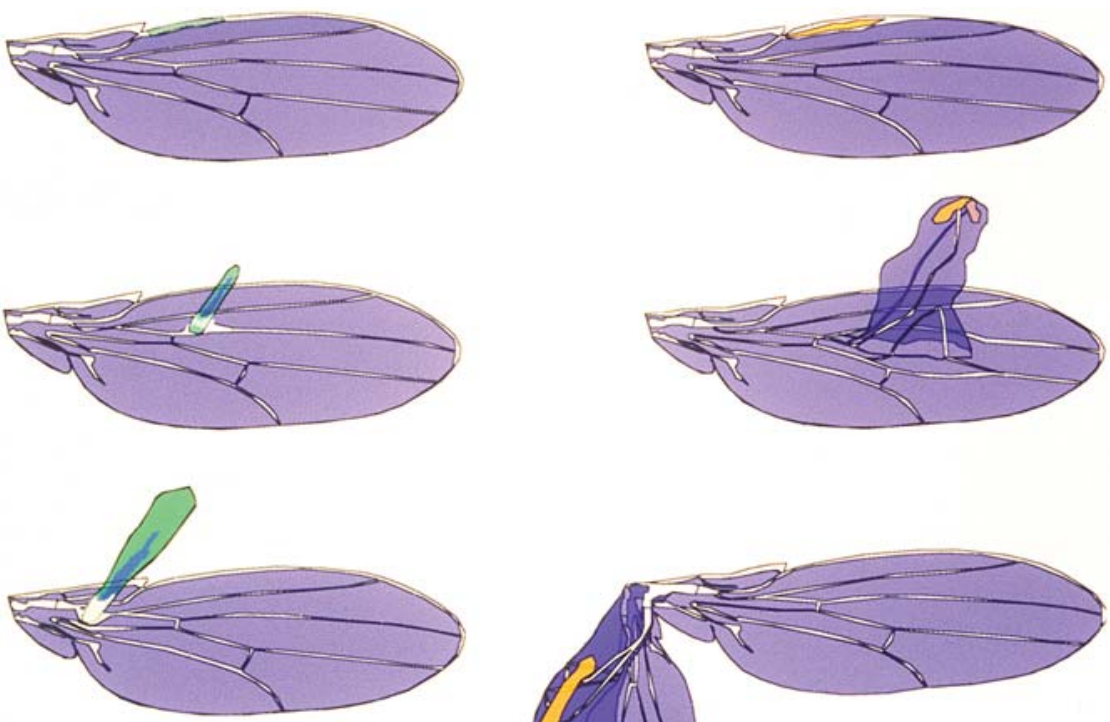

$\mathrm{Vg}$

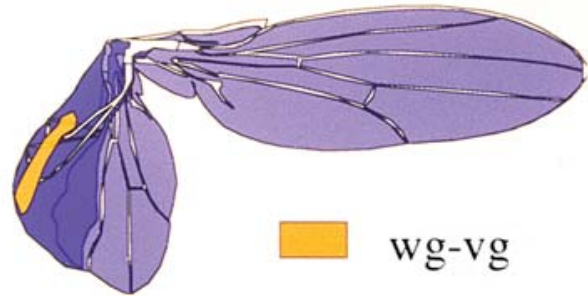

Fig. 16. Scheme of outgrowth resulting from the clonal overexpression of $v g$ alone or in combination with $\mathbf{W g}$, depending on position. Notice the non-autonomous effects outside of the outline of the clones. sponding to more proximal regions but without non-autonomous effects in surrounding wild type cells. The same nub clones initiated in any compartment, in proximal regions, cause nonautonomously a reduction in distal growth of the remaining wing disc in all compartments (negative accommodation) (Fig. 18, Cifuentes and García-Bellido, 1997). This is an example, among others, indicating that A-P and P-D growths are not independent variables. Interestingly, a clonal description of how nub mutant wings grow shows clones with a shape orthogonal to the P-D axis of the wing, i.e. they grow parallel to the margin. Such shape is indicative of cell proliferation hindered in the generation of more distal positional values, hence causing shorter and blunt wings. Moreover, late wild type $\mathrm{M}+$ clones in a $\mathrm{M} / \mathrm{M}+$ background may cross the $D / V$ and $A / P$ compartment boundaries. This is compatible with the notion that the positional values at the borders of nub mutants are still low in these stages allowing $M_{+}$faster growing cells to stride them (Cifuentes and García-Bellido, 1997).

Similar heterogeneities appear along the A-P axis of the wing blade, orthogonally to the wing veins. The latter are defined by clonal restrictions that separate intervein regions in an alternating landscape of crests and valleys (González-Gaitán et al., 1994). Mutant cells of members of the epidermal growth factor receptor pathway, cease to proliferate prior to metamorphosis, leading to normal shape but smaller wings without veins (Garcia-Bellido et al., 1994). Mutant clones grow less, shortening intervein regions and causing negative accommodation of surrounding territories (Fig. 1 in García-Bellido et al., 1994).

Mutations in the extramacrochaetae (emc) gene which encode for a helix-loop-helix (HLH) protein, a negative regulator of basic $\mathrm{HLH}(\mathrm{bHLH})$ proteins, cause narrow clones growing to meet the veins. When they reach two contiguous veins, they prevent the growth of this region, causing negative accommodation in growth of the rest of the wing. The cell-autonomous tendency to grow towards veins indicates that the mutant cells generate high positional values that, when they occupy a whole intervein region prevents its growth. This is possibly the result of homogeneously distributed high positional values in mutant cells (see Fig. 3 in de Celis et al., 1995).

Positive and negative accommodation in mosaics extends its effects to other compart- 
A
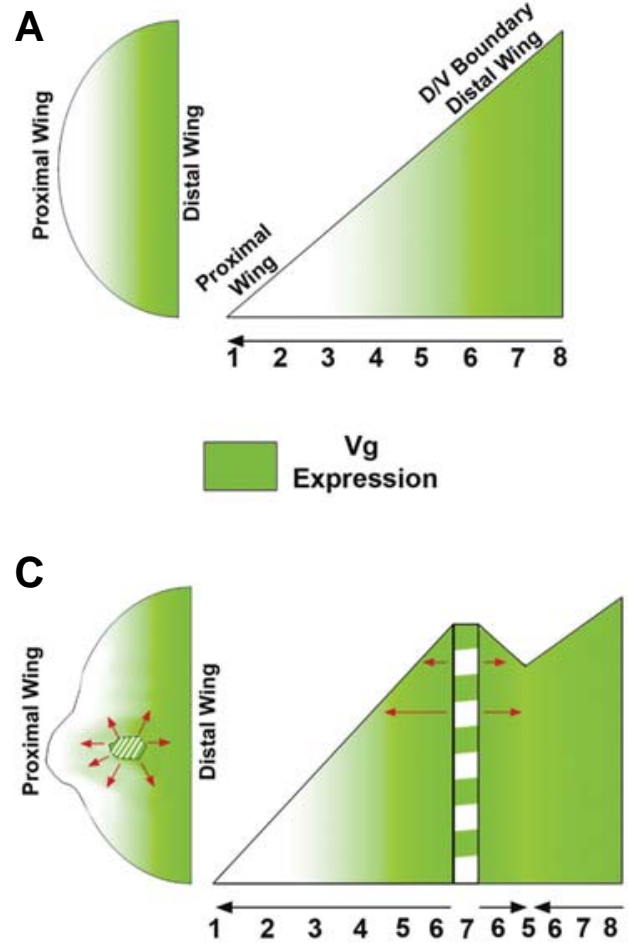

Overexpression clone of $\mathrm{vg}$
B

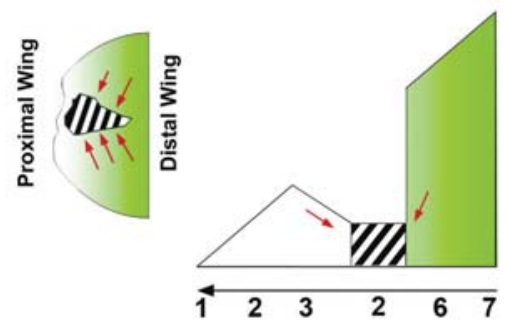

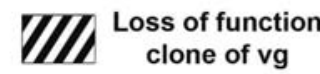

D

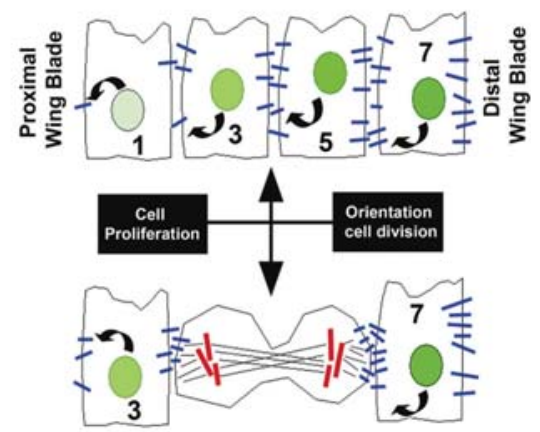

Fig. 17. The internalistic view of organ growth. (A) Vg (green) is heterogeneously distributed along the $P / D$ axis in the wing blade. The decreasing gradient of vg expression from the DN boundary is indicated by lower intensity of green color; numbers correspond to arbitrary positional values in the slope. (B) The loss of function clones of $\mathrm{vg}$ in the wing blade autonomously and non-autonomously reduce growth in the wing blade. The lack of growth is indicated by red arrows. Notice that cells of the wing blade show the scale of positional values diminished and the exchange of positional values between mutant and WT cells is also perturbed, preventing full intercalation. (C) $\mathrm{Vg}$ expressing clones can autonomously and nonautonomously promote cell proliferation in a position dependent manner. The non-autonomous growth and the intercalary cell proliferation in response to vg expressing cells are indicated by red arrows and positional values. (D) Cell proliferation and the orientation of cell divisions depend on differences in vg expression between neighboring cells, rather than on their absolute value. Membrane molecules (blue bars) possibly translate the information of vg expression (positional values) into local cell interactions, driving the rate of cell proliferation and orientation of cell divisions in the wing blade (Baena-López and García-Bellido, 2006). ments. Examples are en (Fig. 11), vg (Fig. 13), nub(Fig. 18) and in mosaics of mutants that cause changes in cell size and cell proliferation rate (Resino and García-Bellido, 2002). Accommodation between mutant and wildtype territories is possibly always reciprocal. Reciprocal accommodations are clear in the confrontation of bithoraxand postbithoraxmutant territories with adjacent wildtype ones across the A/P boundary of halteres (GonzálezGaitán et al., 1990). Along the compartment boundary, the positional values of both regions have to be compressed or expanded in order to obtain the best possible matching. Those behaviors indicate that positional differences and accommodation growth drive cell proliferation beyond compartment boundaries, the source of morphogens. Compartments are then not independent units of growth, as often postulated.

\section{Mitotic orientation in the wing blade}

The direct observation of mitotic figures in certain mutant wings allows us to correlate these orientations with clone shapes and, by extension, adult wing shapes. Thus, the mutant enwings, with posterior compartments as a mirror image duplication of anterior ones, are broader and larger than anterior ones (García-Bellido and Santamaría 1972). A study of how the mutant compartment grows reveals clone shapes similar to those in the anterior compartment (Fig. 16). Thus, the homeotic transformation is associated with changes in mitotic orientations of individual proliferating cells.

The wings (and legs) of some planar polarity mutants (PCP) are rounded and larger such as $d$ sand ft. (review by Zallen 2007). Genetic mosaics of $d s$ show autonomous effects in clones of a rounded shape (Baena-López et al., 2005). An analysis of mitotic orientation in mutant wing blades reveals random orientations. Interestingly, the same occurs in central regions of wings when ds expression is reduced in this territory (Fig. 20). Thus, the normal wild type orientations over the wing result from a fine modulation of $d s$ expression in the membrane of proliferating cells. We find a similar random mitotic orientation in ftmutants. This cell behavior of some PCP mutants in mosaics is reminiscent of that of $v g$ mutants, in accordance with the fact that these genes are controlled by $v g$. However, this is not so in other PCP mutants, which have normal-sized and shaped wings. Probably, the effects in cell

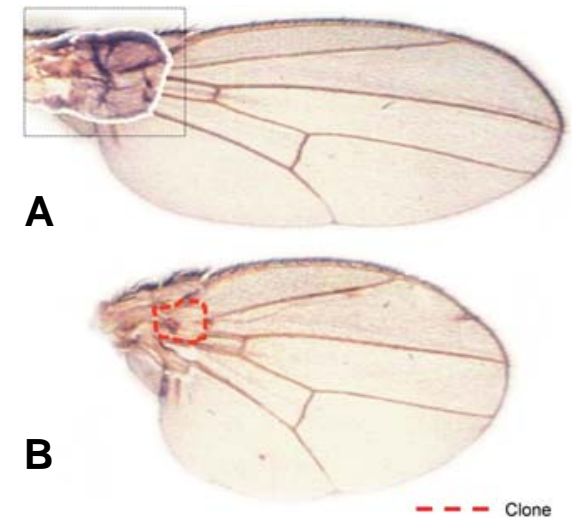

Fig. 18. The adult nub ${ }^{1}$ wing overprinted on the wildtype to show its reduction in size (A). (B) The non-autonomous effects of a nub ${ }^{1}$ clone on an heterozygous nub/+ wing (From Cifuentes and Garcia-Bellido, 1997). 
A

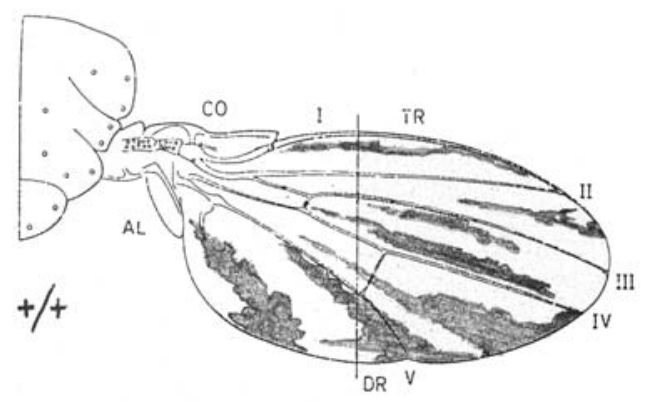

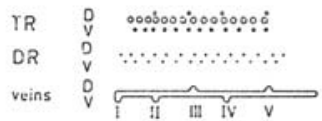

B

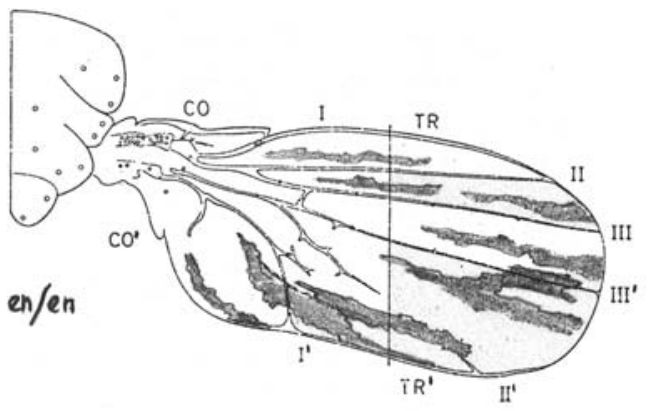

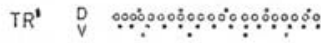

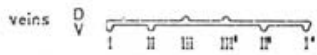

Fig. 19. Clonal growth in the posterior compartment of engrailed mutant wings is like the anterior, wildtype one. Co, costa; TR, triple row; 1-III longitudinal veins, TR, I-III, duplications. Below, the scheme of the TR, DR and vein corrugation along the section of the wing blade (García-Bellido and Santamaría, 1972).

polarity in these mutants are restricted to cuticular cell differentiation stages. Some of them are known to differ in the proximo-distal subcellular localization in the membrane of differentiating cells.

We have seen that wing size changes are associated with mitotic cell polarity in $e n, v g, d s$, ftflies. We have seen, in addition, that clone shape varies with the region of the wing and is different in other appendages, like the legs. Within the wing proper, the prevalent orientations are different along the wing margin, where they are mostly parallel to the margin and in central regions, where they appear orthogonal to it (with occasional A-P cell divisions that determine the width of the clones and the shape of the wing) (Fig. 21). We analyzed the possible role of classical morphogens, such as $\mathrm{Dpp}$ and $\mathrm{Wg}$, in the orientation of cell divisions. In the wing disc margin, $w g$ is expressed in a few cells twins reveals that whereas the twins run P-D, wg blade clones tend to grow parallel even at long distances to the wing margin. The over-expression of $w g$ along the dpp region shows similarly mitotic orientations parallel to the endogenous $w g$ of the wing margin and intermediate angles in the quadrant defined by both endogenous and ectopic wg expressing territories (Fig. 24). Clearly, wg contributes to mitotic orientation of the blade cells. These long-range effects could be explained by diffusion of $w g$ from the margin. We find, however, the same behavior in mutant clones for downstream negative regulators of $w g$ signaling, such as axin, which acts cell-autonomously. Consequently we advance the hypothesis that $w g$ expressing cells determine the perpendicular orientation (P-D) of wing blade $\mathrm{vg}$ dividing cells and vice versa (Baena-López et al., unpublished). This hypothesis
A

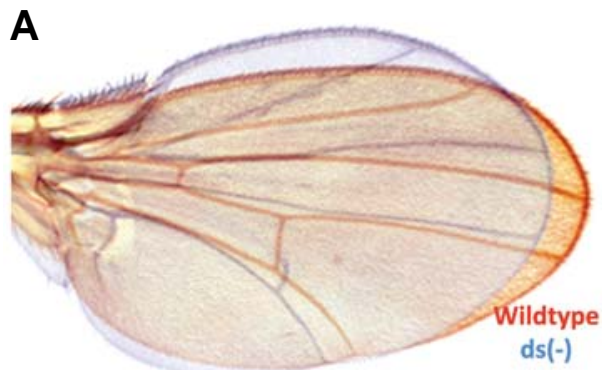

D

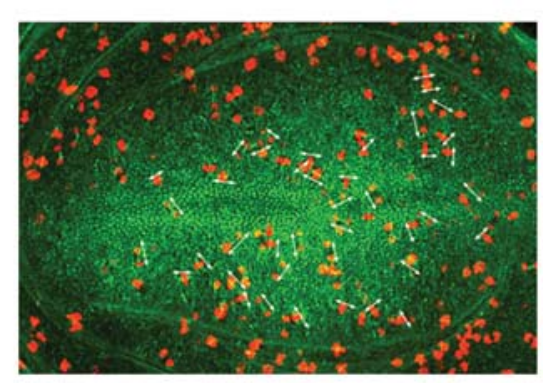

B

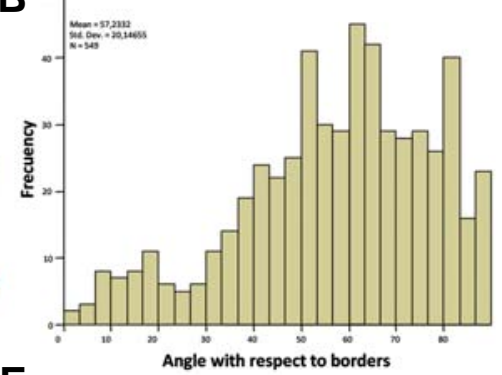

E

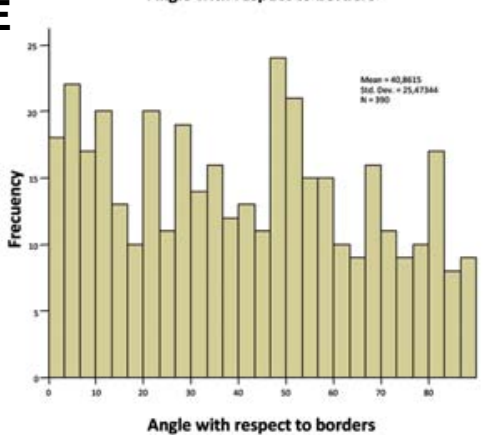

C
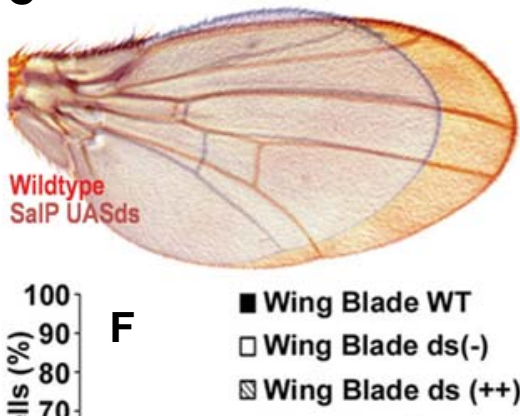

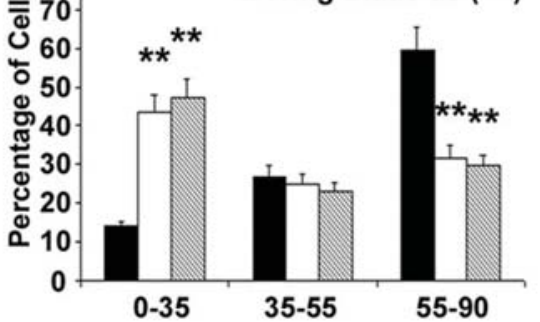

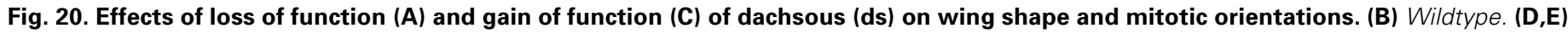
Compare Wildtype with ds(-) and $d s(++)$ in the mitotic orientations (Baena-López et al. 2005). 

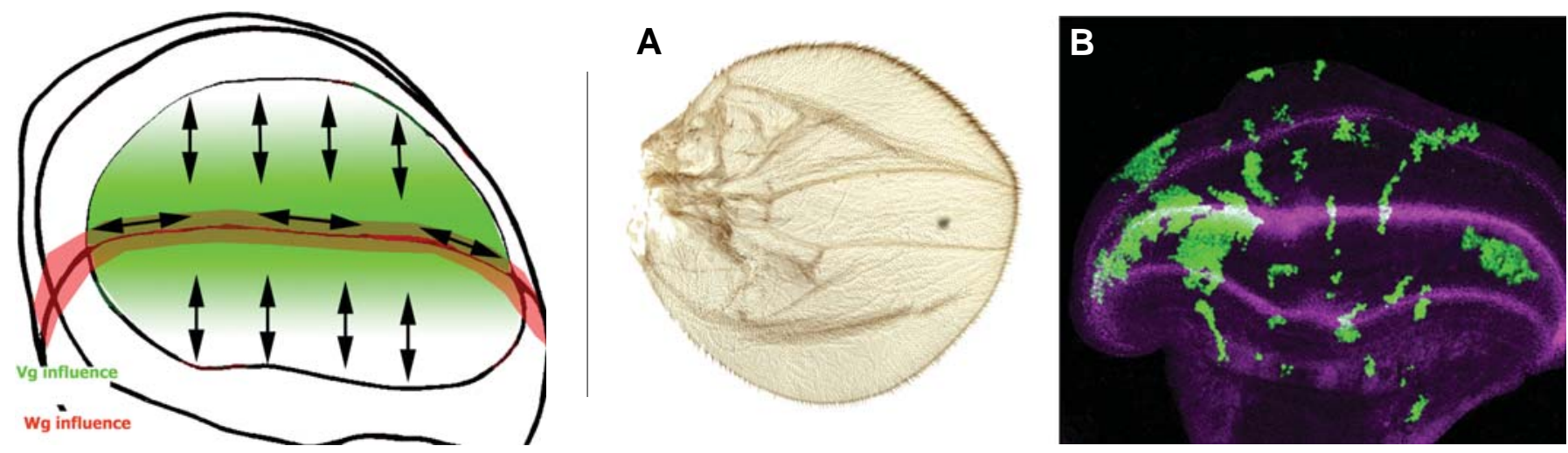

Fig. 21 (Left). Regional distribution of oriented cell divisions in the wing blade in the margin (wg) and in centric regions (vg).

Fig. 22 (Right). Wings with dpp expression along the wing margin (driven by vg-Gal 4) on cell proliferation. (A) Adult wing. (B) Wing disc with flp Lac-Z clones.

can be applied to that of the shape of the wing width, if $w g$ expression in the margin causes the orthogonal orientation of $v g$ cells in the wing blade. Obviously, regional differences in the wing blade cannot be explained by this simple rule. Local modulators must play a role as well. The elongated shape of clones in the legs may result from a prevalent function of a P-D orientation without intervention of cell divisions orthogonal to it.

\section{Size and shape; the questions}

We have discussed above how size and shape in organs result from local cell interaction rather than from the response by the cells to global controls. Hormones and morphogens, in this view, operate merely as growth factors, necessary for cell proliferation and survival but without instructive roles, in a similar way to the effects of metabolites in enzyme induction or antigens determining the specific structure of antibodies.

We have also seen that the size and shape are causally interrelated at the cellular level. How does it all come about? The manifestation of positional information in cells requires two axes of reference and a defined cell polarity. In regeneration and cell re-aggregation experiments, as well as in genetic mosaics, these properties are expressed in the cell membrane. What are the molecular bases of this cell anisotropy? The short answer is that we do not know. We know, at least, that cadherins, as cell adhesion molecules, and tubulins of the spindle and the cytoskeleton must be involved. How their synthesis is quantitatively controlled, or even more, how are these molecules anisotropically assembled and deposited? How is cell polarity determined?

We will actualize here a generative model, the Entelechia model (Fig. 25, García-Bellido and GarcíaBellido 1998), which tries to answer these questions. The Aristotelian word Entelechia denotes the progress of developing organisms to reach their species-specific completion or perfection. Positional differences probably start in the imaginal disc primordium, reflecting heterogeneities in the blastoderm. The gene enis already involved in the generation of the A/P segmental subdivision. The D/
V subdivision appears after some cell divisions as determined by A-P axis and apexpression perpendicular to it. From there the tip of the wing is somewhat defined and subsequent growth with positional value differences in mitotic orientations, ensues. How these cell behavior differences arise is again unknown. Intercalary regeneration and accommodation in mosaics indicate that positional value differences drive proliferation. In this view, cells compute the positional values of their neighbors, compare them with their own values, and when differences are larger than a given threshold or "increment" value (possibly reflecting efficiency in the transmission/perception of signals) cell division takes place. The simplest way of measuring differences between cells is by titration between incoming signals and the own value of the responding cell. However, there is more to it, because the cells orient their division-axis with respect to incoming signals along the positional differences at opposite sides of the cell. The $v g$ gene could correspond to such "own value" gene for P-D growth in the wing blade (a "martial" gene in the original model,
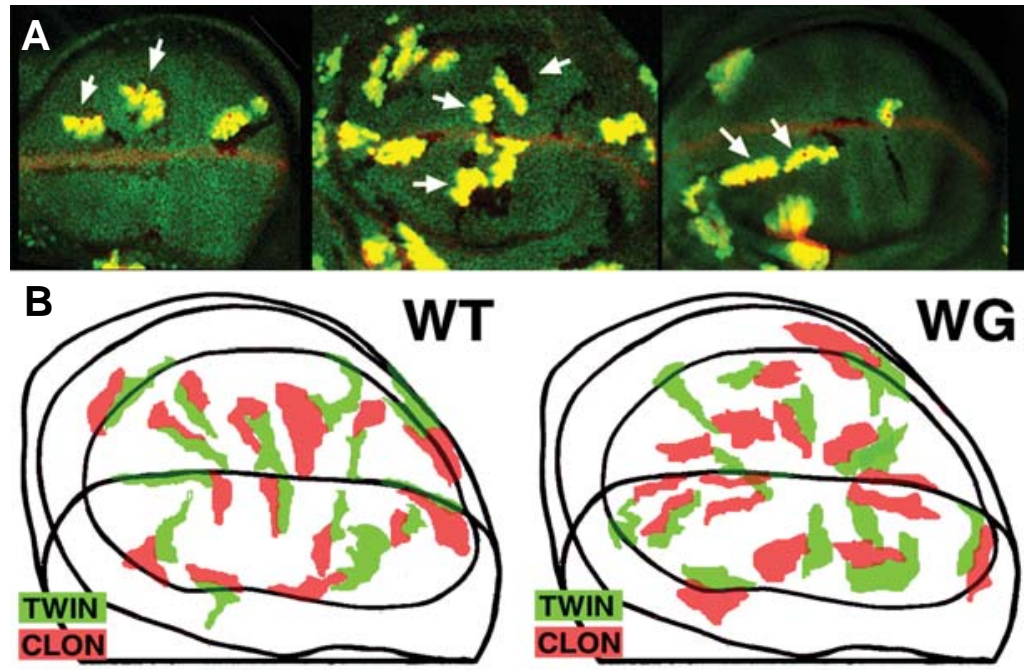

Fig. 23. Clones of $\mathbf{w g}$ expressing cells and their twins in the wing blade. (A) Clones marked by $2 x G F P$ and twins (WT) by OX FGP. (B) Plot of wildtype and wg expressing cells in a diagram. (From L.A. Baena-López and A. Baonza, unpublished). 


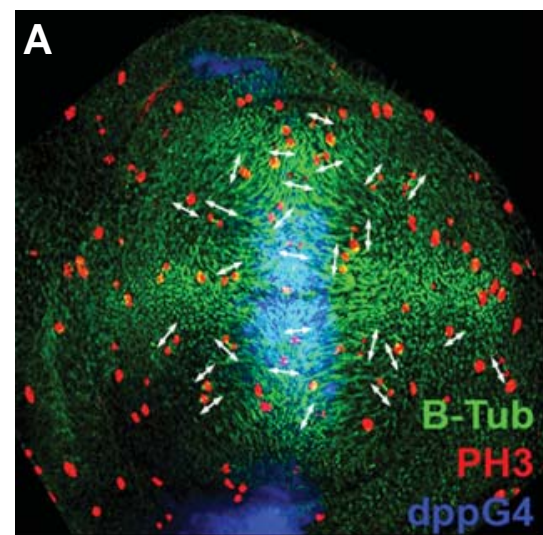

Fig. 24. Ectopic expression of wingless (wg) along the A/P border. (A) Mitotic orientation in wing discs with an overexpression of $w g$ in the dpp region. (dppGal4/UAS wg). (B) Plot of percent of orientation in the dpp domain and outside it. Same symbols as in Fig. 20). (From L.A. Baena-López and A. Baonza, unpublished).

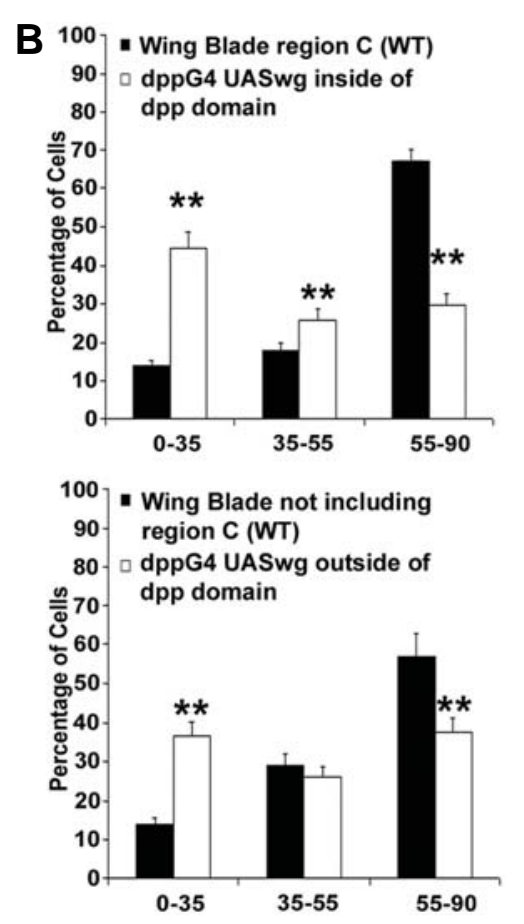

orientated waves?) follows by intercalation of positional values, generating higher values and symmetries in the (clonal borders) and consequently and down slope in all the cells, with new intercalary differences between them. As we have seen growth is intercalary, i.e. cells have to reset there positional values after each cell division, possibly by a positive loop of martial genes activation. To that end, the absolute martial values of the cells must proportionally contribute to the amount of signals sent to neighboring cells. In this operation, receptors, adhesors and ligands must intervene. Postmitotic cells then average their martial value with respect to neighboring ones and wait until the arrival of new incoming signals. Intercalar growth can be affected by genetic or experimental perturbations down the slope of the positional gradient. Such is the case in intercalar regenerations and in $v g$ and nub mosaics. Cell proliferation ceases when the maximal martial values are reached along the axes of reference and the increment values between cells become minimal, i.e. imperceptible by neighboring cells. The organ thus reaches the Entelechia condition.

Graded positional values in the form of amounts of adhesor molecules, activated receptors and their anisotropic distribution in the cell membrane are surely García-Bellido and García-Bellido 1998). Genes like $d s$, ft, and others studied in Resino and García-Bellido (2004) could be involved in the definition of increment values.

In any moment of development, the highest positional values appear in the borders of the primordium, the symmetry borders of the $A-P$ and $D-V$ compartments, and later wing-veins, etc. It is cell proliferation what generates the dimensions of the borders, not the other way around. Progression of cell divisions (associated to operative in normal development. They may explain the outcome of cell recognition in pattern reconstruction in reaggregates and following transdetermination. They may be also operative in terminal and intercalar regeneration, leading to the appearance of new positional values in the cells of the border of the blastema that create higher (or lower) values along the axes of reference (see computer simulations in García-Bellido and García-Bellido 1998). And finally it is operative in feeder layer experiments. Size and
A

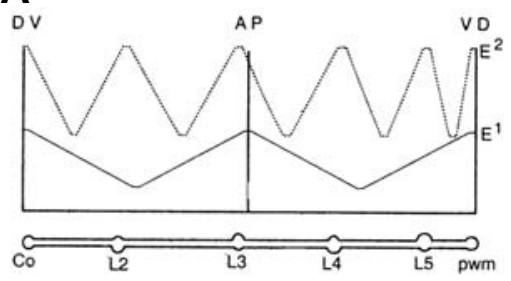

C

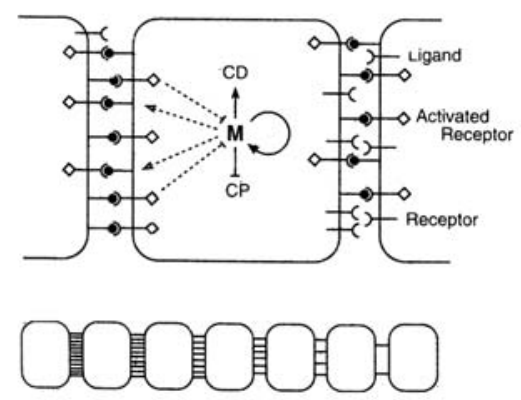

B
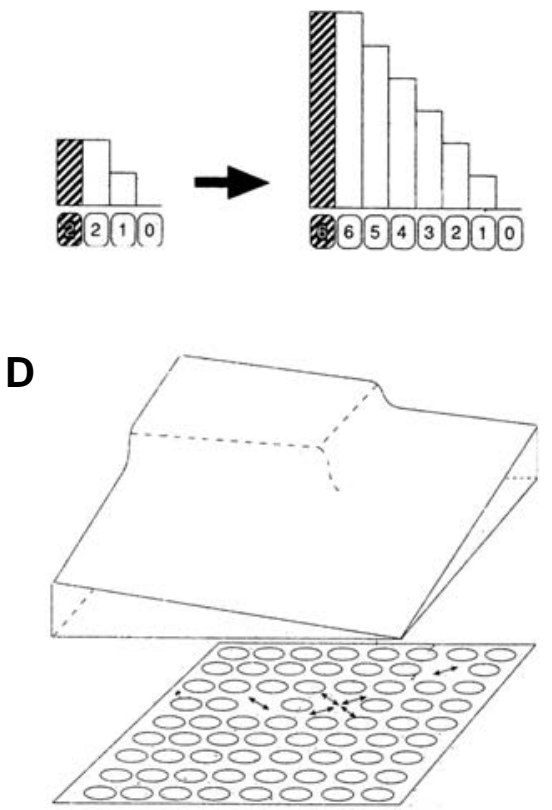

Fig. 25. The Entelechia model for the wing disc. (A) Upper panel, the generative gradients corresponding to the Entelechia condition for compartments (E1) and for vein sectors (E2) in either the dorsal or the ventral surface of the wing. Note that the slopes need not to be the same for each vein sector, as they may depend on local variations of the incremental value for $E s$. A, anterior; $P$, posterior; $D$, dorsal; $V$, ventral. (B) Two stages of the growth in which the increasing amount of martial (M) gene expression (ordinates: amplitude value of $\mathrm{M}$ genes and their ligands) is correlated with position (numbers, correspond to positional values). (C) The components of the Entelechia model, showing how cell communication controls cell division. The activated form of the receptors may be associated with specific adhesion molecules, not shown in the figure. The cell will enter $C D$, cell differentiation, or $C P$, cell proliferation, depending on the balance between $\mathrm{M}$ gene expression and $\mathrm{M}$ gene titration by the activated receptors. Bottom, a row of cells with different amounts of activated receptors, illustrating how the distribution of activated receptors polarizes each cell. (D) Gradients of scalar positional values and scalar discontinuities in the $X$ and $Y$ axes of the epithelium. Cell division (arrows) is elicited whenever discontinuities appear above the threshold for $\mathrm{M}$ disparity detection, at the position where there is a disparity in both $X$ and $Y$ axes. 
shape of a given organ varies among species and higher taxa. This could be caused by small variations in maximal martial or increment values genetically determined.

This model may apply to growth and shape of clones in the wing blade but other Entelechia functions may operate in different organs (as legs) or different regions of the wing such as notum, axillar regions, wing margin, peripodial membrane etc. Reference axes can be very long in number of cells. Possibly, these axes are subdivided later in development into regions with shorter intervals as in leg segments or intervein regions. These regions are not separated by clonal restrictions but could consist of borders between territories of differential gene expression. We do not know how maximal-minimal positional values are defined or what is the nature of the signals and receptors between neighboring cells in these Entelechia functions of long and short wavelengths. Neither do we know how the main orientation of clones, related to shape, which differs among regions, is determined.

The present assay enlightens the problem of the generation of two-dimensional organs, but we are far from expecting that this interpretation can be extended to the generation of 3D organs, such as mesenchyme derivatives, muscles and bones, in Vertebrates. Clearly, the understanding of such classical problems of morphogenesis on the acquisition of constant species-specific size and shape of organs requires much more investigation at the cell biology level. This is the realm of cell interactions in cell populations, and of the dynamics of cell behavior.

\section{Acknowledgements}

I wish to thank the many collaborators of the lab along the years on the study of morphogenesis. In particular, more recently to A. Baena-López and $A$. Baonza that provide me with unpublished results, as well as J.F. de Celis for the constructive criticism to the manuscript. I thank cordially Paloma, Diego and Almudena for their help for they know why. This work was supported by grants from the Dirección General de Investigación Cientifica y Técnica and an institutional grant from Ramón Areces Foundation to the Centro de Biología Molecular "Severo Ochoa".

\section{References}

BAENA-LOPEZ, L. A., BAONZA, A. and GARCIA-BELLIDO, A. (2005). The Orientation of Cell Divisions Determines the Shape of Drosophila Organs. Curr. Biol. 15: 1640-1644.

BAENA-LOPEZ, L.A. and GARCIA-BELLIDO, A. (2006). Control of growth and positional information by the graded vestigial expression pattern in the wing of Drosophila melanogaster. Proc. Nat. Acad. Sci. 103:13734-13739.

BOHN, H. (1970). Interkalare Regeneration und segmentale Gradienten bei den Extremitäten von Leucophaea-Larven (Blattari). I. Femur und Tibia. Wilhelm Roux' Arch. 165: 303-341

CIFUENTES, F. J. and GARCÍA-BELLIDO, A. (1997). Proximo-distal specification in the wing disc of Drosophila by the nubbin gene. Proc. Natt. Acad. Sci. USA. 94: 11405-11410.

DE CELIS, J.F., BAONZA, A. and GARCIA-BELLIDO, A. (1995). Behavior of extramacrochaetae mutant cells in the morphogenesis of the Drosophila wing. Mech. Dev. 53: 209-221.

GARCÍA-ALONSO, L. and GARCÍA-BELLIDO, A. (1988). Extramacrochaetae, a trans-acting gene of the achaete-scute complex of Drosophila involved in cell communication. Wilhelm Roux's Arch. Dev. Biol. 197: 328-338

GARCÍA-BELLIDO, A. (1965). Larvalentwicklung transplantierter Organe von Drosophila melanogasterim Adultmilieu. J. Ins. Physiol. 11: 1071-1078.

GARCÍA-BELLIDO, A. (1966). Pattern reconstruction by dissociated Imaginal Disk cells of Drosophila melanogaster. Dev. Biol. 14: 278-306.

GARCIA-BELLIDO, A. (1966). Changes in selective affinity following transdetermination in imaginal disc cells of Drosophila melanogaster. Exptl. Cell Res., 44: 362-392.

GARCIA-BELLIDO, A. (1972). Pattern Formation in Imaginal Disks. In Results and Problems in Cell Differentiation. Vol. 5, pp.59-91. (H. Ursprung, R. Nöthiger, eds.) Springer-Verlag. Berlin.

GARCÍA-BELLIDO, A., CORTÉS, F. and MILÁN, M. (1994). Cell interactions in the control of size in Drosophila wings. Proc. Natl. Acad. Sci. USA. 91: 1022210226

GARCÍA-BELLIDO, A.C. and GARCÍA-BELLIDO, A. (1998). Cell proliferation in the attainment of constant sizes and shapes: the Entelechia model. Int. J. Dev. Biol. 42: 353-362.

GARCÍA-BELLIDO, A. and MERRIAM, J.R. (1971). Parameters of the Wing Imaginal Disc Development of Drosophila melanogaster. Dev. Biol. 24: 61-87.

GARCÍA-BELLIDO, A. and NÖTHIGER, R. (1976). Maintenance of determination by cells of imaginal discs of Drosophila. Wilhelm Roux's Arch. 180: 189-206.

GARCÍA-BELLIDO, A., RIPOLL, P. and MORATA, G. (1973). Developmental compartmentalization of the wing disk of Drosophila. Nature New Biology245: 251-253.

GARCÍA-BELLIDO, A. and SANTAMARÍA, P. (1972). Developmental analysis of the wing disc in the mutant engrailed of Drosophila melanogaster. Genetics 72: 87-104.

GONZALEZ-GAITAN, M.A., CAPDEVILA, M. P. and GARCIA-BELLIDO, A. (1994). Cell proliferation patterns in the wing imaginal disc of Drosophila. Mech. Dev. 40: $183-200$

GONZALEZ-GAITAN, M.A., MICOL, J.L. and GARCIA-BELLIDO, A. (1990). Developmental genetic analysis of Contrabithorax mutations in Drosophila melanogaster. Genetics 126: 139-155.

HADORN, E. (1978). Transdetermination. In The Genetics and Biology of Drosophila (Eds. M. Ashburner and T.R.F. Wright). Vol. 2C, pp. 556-617. Academic Press.

KERSZBERG, M. and WOLPERT, L. (2007). Specifying positional information in the embryo: looking beyond morphogens. Cel/130:205-209.

MILÁN, M., CAMPUZANO, S. and GARCÍA-BELLIDO, A. (1996). Cell cycling and patterned cell proliferation in the wing primordium of Drosophila. Proc. Nath. Acad. Sci. USA. 93: 640-645

MILÁN, M., CAMPUZANO, S. and GARCíA-BELLIDO, A. (1997). Developmenta parameters of cell death in the wing disc of Drosophila. Proc. Natl. Acad. Sci. USA. 94: 5691-5696.

MIRTH, C.K. and RIDDIFORD, L.M. (2007). Size assessment and growth control: how adult size is determined in insects. Bioessays 29: 344-355.

RESINO, J. and GARCÍA-BELLIDO, A. (2004). Drosophila genetic variants that change cell size and rate of proliferation affect cell communication and hence patterning. Mech. Dev. 121: 351-364.

RESINO, J., SALAMA-COHEN, P. and GARCÍA-BELLIDO, A. (2002). Determining the role of patterned cell proliferation in the shape and size of the Drosophila wing. Proc. Natl. Acad. Sci. USA. 99: 7502-7507.

WOLPERT, L. (1969). Positional information and the spatial pattern of cellular differentiation. J. Theor. Biol. 25: 1-47.

ZALLEN, J. A. (2007). Planar polarity and tissue morphogenesis. Ce//129: 10511063. 


\section{Further Related Reading, published previously in the Int. J. Dev. Biol.}

See our recent Special Issue Epigenetics \& Development edited by Saadi Khochbin and Stefan Nonchev at: http://www.ijdb.ehu.es/web/contents.php?vol=53\&issue=2-3

See Special Issue Pattern Formation edited by Michael K. Richardson and Cheng-Ming Chuong at: http://www.ijdb.ehu.es/web/contents.php?vol=53\&issue=5-6

Ontogeny of an adventurous mind: the origin of Antonio García-Bellido's contributions to developmental genetics

Alain Ghysen

Int. J. Dev. Biol. (2009) 53: 1277-1290 (doi: 10.1387/ijdb.072384ag)

Debatable issues. Interview with L Wolpert and A García-Bellido.

Alain Ghysen

Int. J. Dev. Biol. (1998) 42: 511-518

Syntagms in development and evolution.

\section{F Huang}

Int. J. Dev. Biol. (1998) 42: 487-494

From selectors to realizators.

J Pradel and R A White

Int. J. Dev. Biol. (1998) 42: 417-421

Cell proliferation in the attainment of constant sizes and shapes: the Entelechia model. A C García-Bellido and A García-Bellido

Int. J. Dev. Biol. (1998) 42: 353-362

Compartment boundaries: where, why and how?

$J$ P Vincent

Int. J. Dev. Biol. (1998) 42: 311-315

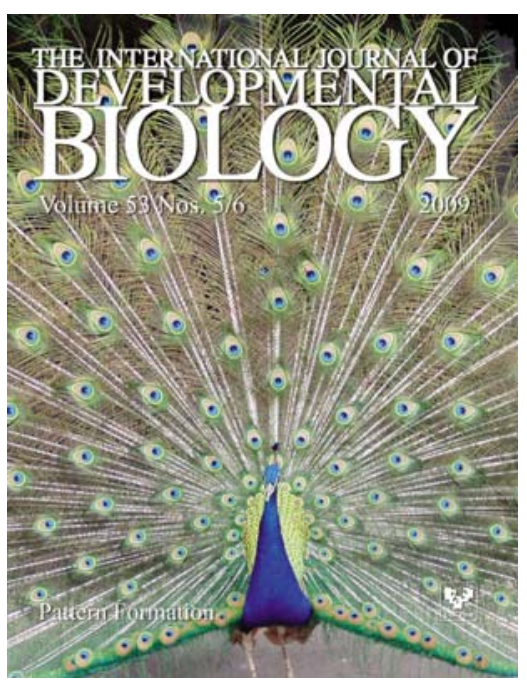

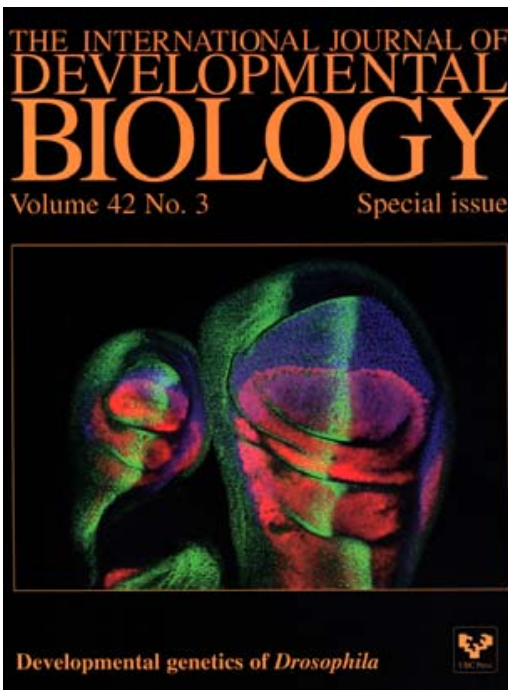

5 yr ISI Impact Factor $(2008)=3.271$

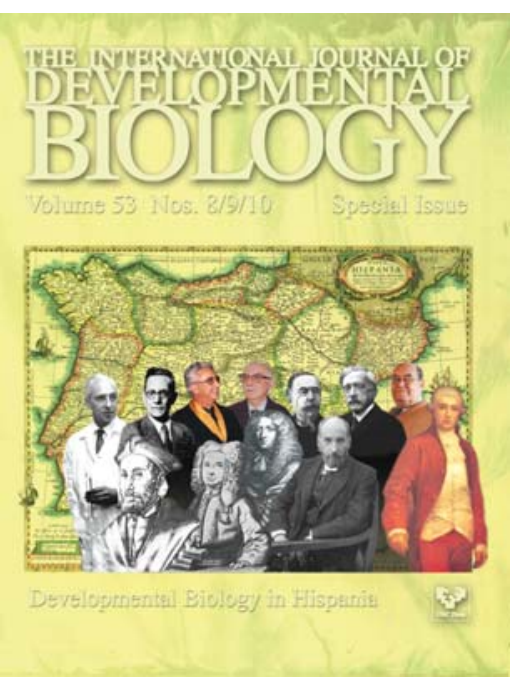

